\title{
Comparison of Gluteus Medius and Tensor Fasciae Latae Muscle Activities during Pilates Clam Exercise Variations
}

\author{
Hyojin Choe', Joong-Hwi Kim,"* \\ ${ }^{1}$ Department of Physical Therapy, Graduate School, Daegu Catholic University, South Korea \\ ${ }^{2}$ Department of Physical Therapy, Daegu Catholic University, South Korea
}

Received April 5, 2021; Revised May 18, 2021; Accepted June 20, 2021

\section{Cite This Paper in the following Citation Styles}

(a): [1] Hyojin Choe, Joong-Hwi Kim, "Comparison of Gluteus Medius and Tensor Fasciae Latae Muscle Activities during Pilates Clam Exercise Variations," International Journal of Human Movement and Sports Sciences, Vol. 9, No. 4, pp. 751 - 756, 2021. DOI: 10.13189/saj.2021.090420.

(b): Hyojin Choe, Joong-Hwi Kim (2021). Comparison of Gluteus Medius and Tensor Fasciae Latae Muscle Activities during Pilates Clam Exercise Variations. International Journal of Human Movement and Sports Sciences, 9(4), 751 756. DOI: 10.13189/saj.2021.090420.

Copyright $\bigcirc 2021$ by authors, all rights reserved. Authors agree that this article remains permanently open access under the terms of the Creative Commons Attribution License 4.0 International License

\begin{abstract}
The purpose of this study was to compare three clam exercises (clam, modified clam 1, and modified clam 2) used in the Pilates physical fitness system, to examine the activity of the gluteus medius and tensor fascia latae and to determine which of the three exercises maximizes the activity of the gluteus medius while minimizing the activity of the tensor fascia latae. Twenty healthy volunteers participated in this study. Surface electromyography (sEMG) was used to measure the electrical activities of the gluteus medius (GMed) and tensor fascia latae (TFL). This study showed significant differences between GMed and TFL muscle activities according to each of the three clam exercises $(p<0.05)$. The gluteus medius showed the highest activity during modified clam 2 and the lowest in clam $(\mathrm{p}<0.05)$. Tensor fascia latae showed the lowest activity during clam and the highest in modified clam $2(p<0.05)$. The gluteus medius to tensor fascia latae ratio was the highest in modified clam 1 and the lowest in modified clam $2(p<0.05)$. Therefore, these findings inform clinicians and Pilates instructors that the appropriate exercise to maximize the activity of the gluteus medius while minimizing the activity of the tensor facia latae is modified clam 1 .
\end{abstract}

Keywords Clam Exercise, Gluteus Medius, Tensor Fasciae Latae

\section{Introduction}

The gluteus medius is a muscle that provides pelvic stability and controls the movement of the thigh while the lower extremities are moving, and the importance of selective strengthening of this muscle in lower and upper musculoskeletal disease exercise programs is constantly emphasized [1-4].

This muscle is the largest of the hip abductor, providing stability of the pelvis and hip joints in the stance phase [5-6].

If the gluteus medius fails to produce normal torque, lateral bending of the trunk and circumduction may occur during walking, and weight support during the stance phase may not be available, producing a Trendelenburg sign in which the pelvis tilts to the opposite side [7-8]. In addition, gluteus medius weakness can cause a number of musculoskeletal disorders, including patellofemoral pain syndrome, iliotibial band syndrome, anterior cruciate ligament sprain, and ankle injury [9-10].

The tensor fasciae latae works with gluteus medius on hip abduction, but also on internal hip rotation [11]. If abnormal alignment of the hip joint occurs due to weakness of the gluteus medius, the knee joint may be moved laterally due to the increased valgus force, resulting in overactivation of the tensor fasciae latae and weakness or contraction of gluteus medius posterior fibers [12]. 
Among the 12 exercises that activate the gluteus medius, the side-lying hip abduction exercise was found to produce the greatest activation [13]. When doing this exercise, there is a need to reduce the compensatory movement of the synergist, quadratus lumborum, and tensor fasciae latae to effectively strengthen the gluteus medius [14]. Therefore, the clam maneuver is an exercise that selectively activates the gluteus medius while minimizing the activity of the tensor fasciae latae [15].

While there have been studies comparing various exercises that strengthen the gluteus medius, there have been none comparing the three clam exercises commonly used in the Pilates exercise program. Therefore, the purpose of this study was to compare the basic clam exercise with two modified forms of the Pilates clam exercises to identify the best one for selectively strengthening the gluteus medius and to present a method that can be applied effectively both in Pilates and rehabilitation exercises.

\section{Materials and methods}

\subsection{Participants}

Twenty healthy individuals volunteered to participate in this study. Participants consisted of 13 adult men and seven adult women in their 20s who are currently attending D University.

The average age of the participants was 24.5 years, the average height $171.15 \mathrm{~cm}$, and the average weight 69.25 kg. Participants were selected among those who had no orthopedic abnormalities in their lumbar or lower extremities in the last six months, had no neurological damage or pain, and had no birth defects.

This study was conducted after obtaining approval from the institutional ethics committee of Daegu Catholic University (CUIRB-2020-0073).

\subsection{Procedures}

Each participant participated in MVIC(maximum voluntary isometric contraction) measurements before performing three clam exercises. The MVIC measurement posture of gluteus medius and tensor fasciae latae was performed in accordance with the standard frequency strength test method of Kendal et al. [11]. Each posture was measured three times for five seconds. By calculating the average value over three seconds, excluding the first and last second, the average value of three repeats was obtained and the \%MVIC was calculated.

Clam (CL), modified clam 1 (M-CL1), and modified clam 2 (M-CL2) were repeated three times each to measure muscle activity. As the three exercises were repeated, the sequence in which they were performed was randomly assigned using lots. Each exercise was maintained for seven seconds, and the measured signals over three seconds were used for analysis, excluding the first and last two second periods. After measuring the muscle activity of each movement, 30 seconds of rest were given to minimize muscle fatigue.

\subsubsection{Clam exercise (CL)}

Each participant is positioned side-lying on a mat. The lower arm extends below the head and alongside the spine. The hip joint bends 60 degrees and the knee joint bends so that the foot is positioned alongside the back of the pelvis. The foot stay dorsiflex. The upper arm is bent and the palm is placed on the mat in front of the chest. With the participant's anterior superior iliac spine (ASIS) facing the front, the upper knee and lower leg to the heel abducts. At this point, linguistic feedback is provided to maximize knee extension to the extent that the pelvis and spine remain motionless.

\subsubsection{Modified clam 1(M-CL 1)}

Each participant is positioned side-lying on the mat. The lower arm extends below the head and alongside the spine. The hip joint bends 60 degrees and the knee joint bends so that the foot is positioned alongside the back of the pelvis. The foot stay dorsiflex. The upper arm is bent and the palm is placed on the mat in front of the chest.

Keeping the femur on the mat, the foot is lifted so that it is aligned with the upper pelvis. At this time, the palm in front of the chest applies force to prevent the body's weight from collapsing forward. With the participant's anterior superior iliac spine (ASIS) facing the front, the upper knee is abducted to the end with the heel attached. At this point, linguistic feedback is provided to maximize knee extension to the extent that the pelvis and spine remain motionless.

\subsubsection{Modified clam 2(M-CL 2)}

Each participant is positioned side-lying on the mat. The lower arm extends below the head and is alongside the spine. The hip joint bends 60 degrees and the knee joint bends so that the foot is positioned alongside the back of the pelvis. The foot maintains dorsiflexion. The upper arm is bent and the palm is placed on the mat in front of the chest. Keeping the femur on the mat, the foot is lifted so that it is aligned with the upper pelvis. At this time, the palm in front of the chest applies force to prevent the body's weight from collapsing forward. With the participant's anterior superior iliac spine (ASIS) facing the front, the upper knee is abducted to the end with the heel attached. At this point, linguistic feedback is provided to maximize knee extension to the extent that the pelvis and spine remain motionless. The upper knee joint is extended while maintaining this position. At this point, the upper foot is plantarflexed.

\subsection{Measurements}

Two electrode pads were attached at $1 / 3$ on the side of 
the greater trochanter of the femur, between the iliac crest and the greater trochanter of femur, to measure EMGs of the gluteus medius.

A further two electrode pads were attached slightly outside and $2 \mathrm{~cm}$ below the anterior superior iliac spine [16], to measure EMGs of the tensor fasciae latae.

All electrode pads were attached by the same experimenter, and skin resistance was minimized by wiping the skin with alcohol cotton and removing hair with a razor. Each electrode pad was attached parallel to myofibres and the distance between the two electrode pads was maintained at approximately $20 \mathrm{~mm}$.

In order to minimize noise, cables for connecting the electrode pad to the EMG equipment used in this study were carefully organized for each experiment. Signals obtained through EMG equipment were collected and analyzed using Laxtha Tesla software 2.0. The surface EMG signal was set to a sampling rate of $1024 \mathrm{~Hz}$. The notch filter was set to $60 \mathrm{~Hz}$ and the bandpass filter was set to $20-500 \mathrm{~Hz}$. The units of all EMG signals were recorded in $\mathrm{mV}$. To reduce the error of the collected EMG signal, the signal-averaged value over three seconds was used and excluded the first two and the last two seconds of the signal. An average was obtained from three repeated measurements. The collected EMG signals were root mean square processed and analyzed.

\subsection{Statistical Analysis}

The statistical program SPSS v18.0 for Windows was used to calculate and compare the mean and standard deviation of the data collected through this study.
One-way repeated measures ANOVA was applied to compare the activity of gluteus medius, tensor fasciae latae, and the ratio of tensor fasciae latae to gluteus medius for the three exercises (CL, M-CL 1, M-CL 2). A post-hoc comparison was performed using Sheffe's method. The statistical significance level $(\alpha)$ was set to 0.05 .

\section{Results}

There were significant differences observed in the activity of the gluteus medius muscle between the three exercises $(p<0.05)$. There was a significant difference in GMed activity between CL and M-CL 1, and a significant difference between CL and M-CL 2, but there was no significant difference between M-CL 1 and M-CL 2.

There was also a significant difference in the activity of tensor fasciae latae between the three exercises $(p<0.05)$. There was a significant difference in TFL activity between CL and M-CL 2, as well as a significant difference between M-CL 1 and M-CL 2, but there was no significant difference between CL and M-CL 1.

There was a significant difference in the activity ratios of the gluteus medius and tensor fasciae latae between the three exercises $(p<0.05)$. Significant differences were seen between CL and M-CL 2, and between M-CL 1 and M-CL 2, but there was no significant difference between CL and M-CL 1.

The closer the activity ratio of GMed-TFL to +1 , the better the exercise and the higher the ratio of GMed to -1 , the higher the activity rate of TFL. The formula for obtaining GMed-TFL ratios is as follows (Table 3).

Table 1. Comparison of gluteus medius muscle activity according to type of exercise

\begin{tabular}{|c|c|c|c|c|c|}
\hline & CL & M-CL 1 & M-CL 2 & $\mathbf{F}$ & $\mathbf{p}$ \\
\hline GMed & $35.1 \pm 23.5^{\mathrm{a}}$ & $56.0 \pm 20.1$ & $60.9 \pm 20.3$ & 8.245 & $0.001^{*}$ \\
\hline \multicolumn{6}{|c|}{${ }^{\mathrm{a}}$ values are mean \pm standard deviation } \\
\hline \multicolumn{6}{|l|}{$\mathrm{p} p<0.05$} \\
\hline \multicolumn{6}{|c|}{ GMed: gluteus medius } \\
\hline \multicolumn{6}{|l|}{ CL: clam } \\
\hline \multicolumn{6}{|c|}{ M-CL 1: modified clam 1} \\
\hline M-CL 2: mo & $\mathrm{m} 2$ & & & & \\
\hline
\end{tabular}

Table 2. Comparison of tensor fascia latae muscle activity according to type of exercise

\begin{tabular}{cccccc}
\hline & CL & M-CL 1 & M-CL 2 & F & p \\
\hline TFL & $13.1 \pm 90.2^{\mathrm{a}}$ & $19.9 \pm 12.9$ & $52.4 \pm 15.9$ & 52.5 & $0.000^{*}$ \\
\hline
\end{tabular}

${ }^{\mathrm{a}}$ values are mean \pm standard deviation

${ }^{*} \mathrm{p}<0.05$

TFL: tensor fasciae latae

CL: clam

M-CL 1: modified clam 1

M-CL 2: modified clam 2 
Table 3. Comparison of GMed-TFL ratio according to sorts of the exercises

\begin{tabular}{lccccc}
\hline & CL & M-CL 1 & M-CL 2 & F & p \\
\hline $\begin{array}{c}\text { GMed } \\
\text {-TFL ratio }\end{array}$ & $0.39 \pm 0.4^{\mathrm{a}}$ & $0.49 \pm 0.2$ & $0.06 \pm 0.3$ & 12.530 & $0.000^{*}$ \\
\hline${ }^{\mathrm{a}}$ values are mean \pm standard deviation & & & \\
${ }^{*}$ p $<0.05$ \\
CL: clam \\
M-CL 1: modified clam 1
\end{tabular}

GMed-TFL ratio $=\frac{\% \text { MVIC of GMed }-\% \text { MVIC of TFL }}{\% \text { MVIC of GMed }+\% \text { MVIC of TFL }}$

\section{Discussion}

This study wanted to compare the relative activities of gluteus medius and tensor fasciae latae muscles during three Pilates clam exercises when conducted on healthy men and women in their 20s.

When exercising to strengthen the gluteus medius, the pelvis can rotate and cause instability, or impair the movement if the synergist, the tensor fasciae latae and quadratus lumborum, make compensatory movements $[14,17]$. In addition, an imbalance in the tensor fasciae latae can increase pressure on the gluteus medius [18]. Therefore, it is important to apply exercise that selectively strengthens just the gluteus medius [19-20]. Several experiments have been conducted on exercises to strengthen this muscle. The ratio of the activity of gluteus medius to tensor fasciae latae showed the greatest activity of the gluteus medius in the clam exercise compared to the other hip abduction exercises. Among the many hip abduction exercises, clam exercises are not the most active for gluteus medius, but are the most appropriate exercises for selective activation [15].

Of the 12 exercises that activate gluteus medius, the side-lying hip abduction exercise proved to be most active [13]. When internal rotation of the hip joint was applied during the side-lying hip abduction exercise, the activity of the tensor fasciae latae was reduced and the gluteus medius was selectively strengthened [21].

Comparing the M-CL2 of the three Pilates clam exercises conducted in this study, the motion is similar to the side-lying hip abduction exercise with hip external rotation, which causes hyperactivity of tensor fasciae latae. However, M-CL2 is a step-by-step movement that transforms the traditional clam exercise, and is not just a movement to abduct the hip joint while the legs are extended, but a movement that extends the knee joint to make the legs longer with the knees abduct, so there is relatively little compensatory movement.

For side-lying hip abduction exercises, early contraction of the quadratus lumborum occurs below 20 degrees [22]. When performing side-lying hip abduction exercises, increasing lumbar stability by using a biofeedback device reduces the activity of the quadratus lumborum and increases the activity of the gluteus medius [23]. Based on the principle of leverage, the three Pilates clam exercises conducted in this study are less compensatory because the action point is close relative to the side-lying hip abduction. Furthermore, the exercises of this study excluded compensatory movements of the spine and pelvis through verbal feedback instead of by using tools to provide stability, and induced only movement of the hip joint.

In this study, we implement modified clam exercises that can provide resistance without using devices for resistance. The modified clam exercises move the weight forward, resulting in greater resistance to the knee and hip joints than the basic clam exercise. As a result, the EMG activity of gluteus medius and tensor fasciae latae in the modified clam exercises compared to the basic clam exercise is higher.

Our data shows that the ratio of the activity of gluteus medius in M-CL 1 is the largest. Therefore, the results obtained are considered to be grounds for selective use of the exercise if the tensor fasciae latae is hyperactive due to deformation of the alignment of the lower extremities or functional disability.

However, this study has clinical limitations in terms of generalizability because the participating subjects were limited to healthy men and women in their 20s. Furthermore, since the results may vary depending on the alignment of the lower extremities, it is suggested that the alignment of lower extremities should also be considered in future studies.

\section{Conclusions}

Twenty healthy men and women in their 20s were compared for the activity of their gluteus medius and tensor fasciae latae muscles in three Pilates clam exercises, with the aim of minimizing the activity of tensor fasciae latae while maximizing the activity of the gluteus medius. EMG pads were attached to the two muscles, and data recorded using the EMG device as three exercises were performed.

The study found that both CL, M-CL 1, and M-CL 2 
were more active in gluteus medius than in tensor fasciae latae, and for gluteus medius, larger activations in the M-CL 1 and M-CL 2 were shown to be significant. In the case of tensor fasciae latae, the largest activation seen with M-CL 2 was significantly greater than with $\mathrm{CL}$ and M-CL 1.

Therefore, to selectively strengthen the gluteus medius, M-CL 1 with a higher gluteus medius activation ratio is more appropriate than M-CL 2, in which the tensor fasciae latae is highly activated.

It is expected that data from this study will help selectively apply the correct exercise according to the alignment of the lower and upper extremities in Pilates training or in rehabilitation programs that enhance gluteus medius.

\section{Acknowledgements}

This work was supported by the sabbatical research grant from Daegu Catholic University in 2019.

\section{REFERENCES}

[1] Earl J. E., "Gluteus medius activity during 3 variations of isometric single-leg stance," Journal of Sport Rehabilitation, vol. 14, no. 1, pp. 1-11, 2005. DOI: 10.1123/jsr.14.1.1

[2] Fredericson M., Cookingham C. L., Chaudhari A. M., Dowdell B. C., Oestreicher N., Sahrmann S. A., "Hip abductor weakness in distance runners with iliotibial band syndrome," Clinical Journal of Sport Medicine, vol. 10, no. 3, pp. 169-175, 2000. DOI: 10.1097/00042752-200007000-00 004

[3] Schmitz R. J., Riemann B. L., Thompson T., "Gluteus medius activity during isometric closed-chain hip rotation," Journal of Sport Rehabilitation, vol. 11, no. 3, pp. 179-188, 2002. DOI: $10.1123 /$ jsr.11.3.179

[4] Sulaiman W., Jumintono, Wahyuniati C., Suryandartiwi W., Sunardi J., Umar F., Manoppo F., Yudanto, Hos J., "Quickly Understanding on Progressive Muscle Relaxation with Video-based Learning in Secondary School Students," International Journal of Human Movement and Sports Sciences, vol. 8, no. 5, pp. 181-185, 2020. DOI: 10.13189/saj.2020.080504

[5] Scholtes S. A., Norton B. J., Lang C. E., Van Dillen L. R., "The effect of within-session instruction on lumbopelvic motion during a lower limb movement in people with and people without low back pain," Manual Therapy, vol. 15, no. 5, pp. 496-501, 2010. DOI: 10.1016/j.math.2010.05.003

[6] Al-Hayani A., "The functional anatomy of hip abductors," Folia Morphol, vol. 68, no. 2, pp. 98-103, 2009. https://journals.viamedica.pl/folia_morphologica/article/vie w/15935

[7] Granat M. H., Maxwell D. J., Ferguson A. C., Lees K. R., Barbenet J. C., "Peroneal stimulator: Evaluation for the correction of spastic drop foot in hemiplegia," Archives of
Physical Medicine and Rehabilitation, vol. 77, no. 1, pp. 19-24, 1996. DOI: 10.1016/s0003-9993(96)90214-2

[8] Daskalaki K., Pafis G., Gioftsidou A., Beneka A., Bebetsos E., Malliou P., "Investigation of the effects of leg dominance on cross-transfer of flexibility after a unilateral treatment with foam roller-A pilot study," International Journal of Human Movement and Sports Sciences, vol. 8, no. 3, pp. 79-85, 2020. DOI: 10.13189/saj.2020.080301

[9] Nadler S. F., Malanga G. A., Bartoli L. A., Feinberg J. H., Prybicien M., DePrince M., "Hip muscle imbalance and low back pain in athletes: Influence of core strengthening," Medicine \& Science in Sports \& Exercise, vol. 34, no. 1, pp. 9-16, 2002. DOI: 10.1097/00005768-200201000-00003

[10] Niemuth P. E., Johnson R. J., Myers M. J., Thieman T. J., "Hip muscle weakness and overuse injuries in recreational runners," Clinical Journal of Sport Medicine, vol. 15, no. 1, pp. 14-21, 2005. DOI: 10.1097/00042752-200501000-00004

[11] Kendall F. P., McCreary E. K., Provance P., Rodgers M., Romani W., "Lower Extremity," in Muscles: Testing and function, with posture and pain, (5th ed), Lippincott Williams \& Wilkins, 2005. pp. 359-464.

[12] Earl J. E., Hoch A. Z., “A proximal strengthening program improves pain, function, and biomechanics in women with patellofemoral pain syndrome," The American Journal of Sports Medicine, vol. 39, no. 1, pp. 154-163, 2011. DOI: $10.1177 / 0363546510379967$

[13] Distefano L. J., Blackburn J. T., Marshall S. W., Padua D. A., "Gluteal muscle activation during common therapeutic exercises," Journal of Orthopaedic \& Sports Physical Therapy, vol. 39, no. 7, pp. 532-540, 2009. DOI: 10.2519/jospt.2009.2796

[14] Comerford M. J., Mottram S. L., "Movement and stability dysfunction-contemporary developments," Manual Therapy, vol. 6 , no. 1, pp. 15-26, 2001. DOI: 10.1054/math.2000.0388

[15] Selkowitz D. M., Beneck G. J., Powers C. M., "Which exercises target the gluteal muscles while minimizing activation of the tensor fascia lata? electromyographic assessment using fine-wire electrodes," Journal of Orthopaedic \& Sports Physical Therapy, vol. 43, no. 2, pp. 54-64, 2013. DOI: 10.2519/jospt.2013.4116

[16] Criswell E., "The basics of surface electromyography," in Cram's introduction to surface electromyography, (2nd ed), Jones \& Bartlett Publishers, 2010, pp. 1-170.

[17] Sahrmann S., Azevedo D. C., Van Dillen L., "Diagnosis and treatment of movement system impairment syndromes," Brazilian Journal of Physical Therapy, vol. 21, no. 6, pp. 391-399, 2017. DOI: 10.1016/j.bjpt.2017.08.001

[18] McNeill W., Scott S., "Treatment of hip microinstability and gluteal tendinopathies involves movement control and exercise," Journal of Bodywork and Movement Therapies, vol. 20, no. 3, pp. 588-594, 2016. DOI: 10.1016/j.jbmt.2016.06.012

[19] Leon Chaitow N., "MET and the treatment of Joints," in Muscle Energy Techniques, (4th ed), Elsevier Health Sciences, 2013, pp. 164-220.

[20] Richardson C. A., Snijders C. J., Hides J. A., Damen L., Pas M. S., Storm J., "The relation between the transversus abdominis muscles, sacroiliac joint mechanics, and low back 
pain," Spine, vol. 27, no. 4, pp. 399-405, 2002. DOI: 10.1097/00007632-200202150-00015

[21] Lee J., Cynn H., Kwon O., Yi C., Yoon T., Choi W., Choi S., "Different hip rotations influence hip abductor muscles activity during isometric side-lying hip abduction in subjects with gluteus medius weakness," Journal of Electromyography and Kinesiology, vol. 24, no. 2, pp. 318-324, 2014. DOI: 10.1016/j.jelekin.2014.01.008
[22] Frank C., Page P., Lardner R., "Evaluation of Movement Patterns," in Assessment and treatment of muscle imbalance: The janda approach, Human kinetics, 2009, pp. 77-92.

[23] Cynn H., Oh J., Kwon O., Yi C., "Effects of lumbar stabilization using a pressure biofeedback unit on muscle activity and lateral pelvic tilt during hip abduction in sidelying," Arch Phys Med Rehabil, vol. 87, no. 11, pp. 1454-1458, 2006. DOI: 10.1016/j.apmr.2006.08.327 